\title{
INFLUENCE DU STADE DE VÉGÊTATION ET DE L'ESPẼCE VÉGÉTALE SUR LE COMPORTEMENT ALIMENTAIRE ET MÉRYCIQUE DE MOUTONS RECEVANT DES FOURRAGES VERTS HACHÉS
}

\author{
J.-P. DULPHY et G. BECHET \\ avec la collaboration technique de J.-M. Borssav, II. Bousguet et L. L'Hotelier \\ Laboratoire des aliments, \\ Centre de Recherches de Clermont licrand, I. N. R. A., \\ Theix, 63110 Beaumont
}

\section{RÉSUMÉ}

I. Nous avons complété les obscrvations faites par Dulpiry et DemarQuldy (I974) en étudiant l'influence du stade de végétation et de l'espèce végétale sur le comportement alimentaire. et mérycique de moutons recevant, à l'auge, des fourrages verts hachés. Cette étude a porté sur $8+$ échantillons de fourrages verts clont 62 correspondant à 4 espèces végétales suivies systématiquement au cours de leurs différents cycles de croissance.

2. La quantité de matière sèche ingérée lors de chacun des 2 repas suivant la distribution de fourrage aux animaux représente un pourcentage très variable (25 à 4.5 p. 100$)$ des quantités ingérées par jour, mais en valeur absolue les quantités ingérées lors de ces grands repas varient peu en fonction du stade de végétation du fourrage, c'est-à-dire de sa digestibilité, de son ingestibilité et de sa composition chimique. Ces quantités ingérées sont beaucoup plus élevées pour les légumineuses que pour les graminées.

3. Lorsque la plante vieillit la cluréc journalière d'ingestion du mouton diminue généralement: le nombre de repas par jour climinue également nettement, alors que la durée unitaire d'ingestion reste constante ou augmente.

4. La durée journalière de rumination augmente rapidement lorsque la digestibilité de la plante diminue de 80 à 73 p. roo environ. En dessous d'une digestibilité de 73 p. Ioo, la durée de rumination augmente beaucoup plus lentement et ne semble pas pouvoir dépasser 600 minutes par jour chez le mouton. Simultanément la duréc unitaire de rumination augmente nettement lorsque la digestibilité de la plante diminue.

5. La durée journalière de mastication varie peu lorsque la plante vieillit. Par contre la durée unitaire de mastication DUM augmente rapirlement, en particulier lorsque les quantités ingérées par jour QI diminuent :

$$
\left(\mathrm{DUUM}=26,68-2,07 \mathrm{QI} ; \quad \gamma=-0,923^{* *}\right)
$$

6. Les différences observées entre espèces végétales sont parfois importantes, mais elles semblent explicables par les différences de digestibilité ou de teneurs en matière sèche, matières azotées et cellulose brute existant entre plantes. 


\section{INTRODUCTION}

Dans une première étude (DulPhy et Demarquilly, I974), nous avons observé que le mouton recevant du fourrage vert modifiait son comportement alimentaire et mérycique au fur et à mesure du vieillissement de la plante, notamment au cours du premier cycle de végétation. C'est ainsi que le nombre de repas diminue et que la durée journalière de rumination augmente jusqu'à un maximum d'environ Io heures ; on observe aussi une augmentation nette de la durée unitaire de rumination et surtout de la durée unitaire de mastication.

Cependant, cette étude avait été faite avec un nombre limité ( 2 à 4 ) de moutons et les enregistrements des manifestations du comportement n'avaient pas été effectués d'une manière continue au cours des différents cycles de croissance du fourrage.

Nous avons donc voulu confirmer les relations mises précédemment en évidence en travaillant sur un nombre plus important de moutons suivis en permanence et compléter nos observations en mesurant les quantités ingérées lors du grand repas suivant chacune des deux distributions journalières de fourrage afin d'étudier les conditions dans lesquelles s'établit le rassasiement des animaux. Comme dans l'étude précédente il s'agit pour nous d'une étude descriptive permettant de connaître le "comportement de référence " des moutons pour les fourrages verts en en précisant les facteurs de variation les plus importants de façon à avoir une comparaison avec ce qui se passe avec les ensilages (Dur,PHy et DEMARQUIL,LY, I973).

\section{MATÉRIËL ETT MÉTHODES}

De I97I à I973, les manifestations du comportement alimentaire et mérycique des moutons ont été enregistrées pour 84 échantillons de fourrages verts. Parmi ceux-ci, 62 correspondent à 4 espèces végétales : brome (variété $F G 90$ ), ray-grass italien (variété Tétrone) ray-grass anglais (variété Reveille) et trèfle violet (variété Goliath) que nous avons étudiées systématiquement au cours de leurs différents cycles de croissance. Les 22 autres échantillons appartiennent à des espèces végétales diverses (fétuque élevée, fléole, ray-grass italien, luzerne et sainfoin) récoltées à un moment précis (vers le début de l'épiaison) et ne seront cités que par comparaison avec le premier groupe d'échantillons.

Les manifestations du comportement alimentaire et mérycique ont été enregistrées selon la technique de Ruckebusch (r963), pendant 5 jours consécutifs, soit au cours de semaines successives lors de l'étude d'un cycle de végétation, soit après une mise en régime de ro jours environ pour les échantillons de fourrage isolés $(n=22)$. Simultanément la digestibilité et l'ingestibilité (i.e. la quantité volontairement ingérée) étaient mesurées.

Les moutons utilisés étaient des béliers mâles castrés de race Texel âgés de $\mathbf{1} 8$ mois à 3 ans et pesant de 45 à $70 \mathrm{~kg}$. Chaque lot était composé de 6 animaux ; ces animaux étaient toujours les mêmes pour l'étude d'une espèce végétale au cours de ses différents cycles de croissance.

Les fourrages verts ont été fauchés chaque matin, hachés dans un hache-paille (brins de 2 à $3 \mathrm{~cm}$ ) et distribués ad libitum (Io p. roo de refus) en 2 repas par jour à 8 et à I 6 heures; les quantités offertes et refusées ont été pesées chaque jour. De plus, pour 50 échantillons, les quantités de fourrage non consommées ont également été pesées à la fin du repas principal suivant les 2 distributions journalières de fourrage puis remises à la disposition de l'animal.

Les définitions des différents paramètres ont été données par Dulpry (I97I). Les durées journalières d'ingestion et de rumination correspondent à la somme de toutes les activités d'ingestion, ou de rumination durant 24 heures. L'activité de mastication représente l'ensemble des activités d'ingestion et de rumination. Les durées unitaires d'ingestion et de rumination corres- 
pondent au temps passé à ingérer ou à ruminer par gramme de matière sèche ingéré par kilogramme de poids métabolique; ces durées varient donc en sens inverse des vitesses d'ingestion ou de rumination. Nous avons appelé repas (ou période de rumination) une période d'ingestion (ou de rumination) d'une durée au moins égale ou supérieure à 7 minutes. Dès qu'une interruption ci'activité à l'intérieur d'une période d'activité donnée excède 7 minutes, deux périodes sont comptées.

\section{RÉSULTATS}

\section{Composition chimique et digestibilité des fourrages étudies}

Les teneurs en matière sèche, matières azotées totales, cellulose brute, ainsi que 1a digestibilité de la matière organique des différents fourrages sont rapportées dans le tableau I. La composition des plantes en matières azotées est très variable : de I8-20 p. IOo pour les luzernes ou les graminées au début d'un cycle à $6-8 \mathrm{p}$. Ioo pour les graminées à la fin du premier cycle de végétation. Simultanément nous observons des teneurs en cellulose brute allant de I8-2I p. Ioo à 30-34 p. IOO. La digestibilité de la matière organique des différents échantillons varie, quant à elle, de $75-80$ à $60-65$ p. 100.

\section{TABLEAU I}

\section{Composition chimique et digestibilité des fourrages etudiés}

(valeurs moyennes et extrêmes)

\begin{tabular}{|c|c|c|c|c|c|c|}
\hline & & $n$ & $\begin{array}{l}\text { Teneur } \\
\text { en MS } \\
(\%)\end{array}$ & $\begin{array}{c}\text { Teneur } \\
\text { en MA dans la MS } \\
(\%)\end{array}$ & $\begin{array}{c}\text { Teneur en cellulose } \\
\text { brute dans la MS } \\
(\%)\end{array}$ & $\begin{array}{l}\text { Digestibilité } \\
\text { de la MO } \\
(\%)\end{array}$ \\
\hline Brome & $\begin{array}{l}1^{\text {er }} \text { cycle } \\
2^{\mathrm{e}} \text { cycle } \\
3^{\mathrm{e}} \text { cycle }\end{array}$ & $\begin{array}{r}13 \\
9 \\
8\end{array}$ & $\begin{array}{ll}20,4 & (15,9-26,3) \\
23,9 & (20,0-31,8) \\
18,5 & (15,8-21,7)\end{array}$ & $\begin{array}{rr}10,4 & (5,7-15,2) \\
9,8 & (7,5-13,5) \\
14,9 & (13,2-19,2)\end{array}$ & $\begin{array}{ll}28,5 & (21,9-31,5) \\
31,0 & (28,8-32,9) \\
25,5 & (19,6-28,1)\end{array}$ & $\begin{array}{ll}72,0 & (67,0-78,6) \\
67,2 & (60,8-73,9) \\
74,8 & (69,9-83,8)\end{array}$ \\
\hline $\begin{array}{l}\text { Fétuque } \\
\text { élevée }\end{array}$ & $\begin{array}{l}\text { 1er cycle } \\
\text { 2e cycle }\end{array}$ & $\begin{array}{l}7 \\
1\end{array}$ & $\begin{array}{c}17,3(15,8-19,7) \\
21,1\end{array}$ & $\begin{array}{c}12,6(10,9-15,5) \\
13,2\end{array}$ & $\begin{array}{c}28,5 \quad(25,0-32,7) \\
26,3\end{array}$ & $\begin{array}{c}67,3(61,9-70,4) \\
65,0\end{array}$ \\
\hline Fléole & $\begin{array}{l}\text { 1er cycle } \\
2 \mathrm{e} \text { cycle }\end{array}$ & $\begin{array}{l}3 \\
1\end{array}$ & $\begin{array}{c}17,7(15,9-19,0) \\
18,2\end{array}$ & $\begin{array}{c}10,5(10,1-10,9) \\
13,6\end{array}$ & $\begin{array}{c}30,1 \quad(26,7-33,8) \\
28,0\end{array}$ & $\begin{array}{c}68,3(66,5-69,5) \\
68,0\end{array}$ \\
\hline $\begin{array}{c}\text { Ray-grass } \\
\text { italien }\end{array}$ & $\begin{array}{l}1^{\text {er }} \text { cycle } \\
2^{\text {e }} \text { cycle }\end{array}$ & $\begin{array}{l}9 \\
5\end{array}$ & $\begin{array}{l}16,2 \quad(12,2-24,8) \\
18,2 \quad(15,1-23,9)\end{array}$ & $\begin{array}{ll}10,6 & (6,3-18,5) \\
11,2 & (6,7-14,6)\end{array}$ & $\begin{array}{ll}25,5 & (20,3-30,9) \\
28,8 & (26,3-32,0)\end{array}$ & $\begin{array}{ll}71,8 & (63,9-79,6) \\
63,4 & (59,2-69,1)\end{array}$ \\
\hline $\begin{array}{c}\text { Ray-grass } \\
\text { anglais }\end{array}$ & $\begin{array}{l}\text { 1er cycle } \\
2^{e} \text { cycle }\end{array}$ & $\begin{array}{l}6 \\
4\end{array}$ & $\begin{array}{l}16,3(15,1-19,0) \\
16,2(14,3-18,1)\end{array}$ & $\begin{array}{lr}12,6 & (9,7-16,4) \\
13,7 & (11,8-16,2)\end{array}$ & $\begin{array}{ll}2 t^{\prime}, 1 & (19,8-28,1) \\
26,,_{4}^{\prime} & (24,0-30,3)\end{array}$ & $\begin{array}{l}74,3 \quad(67,3-79,0) \\
72,0 \quad(67,6-74,9)\end{array}$ \\
\hline $\begin{array}{l}\text { Trèfle } \\
\text { violet }\end{array}$ & $\begin{array}{l}\text { 1er cycle } \\
2 \text { e cycle }\end{array}$ & $\begin{array}{l}7 \\
4\end{array}$ & $\begin{array}{ll}13,8 & (11,2-16,9) \\
15,9 & (13,6-18,4)\end{array}$ & $\begin{array}{l}15,0 \quad(12,8-18,5) \\
17,6 \quad(16,5-18,5)\end{array}$ & $\begin{array}{ll}2 t_{4}^{\prime} t_{t} & (17,5-29,8) \\
25,2 & (23,5-27,1)\end{array}$ & $\begin{array}{l}70,6(62,5-80,2) \\
68,4 \quad(63,6-73,2)\end{array}$ \\
\hline Luzerne & & 5 & $21,8 \quad(20,4-23,6)$ & $18,7 \quad(16,8-20,3)$ & $27,2(2,+2-30,3)$ & $62,6(58,3-64,6)$ \\
\hline Sainfoin & & 2 & 13,1 et 15,5 & 15,3 et 15,7 & $20,,^{\prime}$ et 23,0 & 68,2 et 70,0 \\
\hline
\end{tabular}


Quantités ingérées (tabl. 2)

Quantité ingérée par jour.

L'ingestibilité des fourrages étudiés est très variable selon l'espèce végétale et le stade de végétation: elle diminue de 70-90 $\mathrm{g}$ de matière sèche par $\mathrm{kg} \mathrm{P}^{\mathbf{0}, 75}$ au début du cycle de végétation à $45-55 \mathrm{~g}$ à la fin du cycle ; pour une plante donnée, elle évolue dans le même sens que la digestibilité de la matière organique et que la teneur en matières azotées et en sens inverse de la teneur en cellulose brute. L'ingestibilité de la luzerne et du sainfoin est très élevée $\left(80\right.$ à $\left.90 \mathrm{~g} / \mathrm{kg} \mathrm{P}^{\mathbf{0}, 75}\right)$ et supérieure $(\mathrm{P}<0,05)$ à celle des graminées de même digestibilité. Par contre celle du trèfle n'est supérieure qu'à celle du brome après la montaison et qu'à celle de la fétuque tout au long du cycle. Les ingestibilités des différentes graminées sont comparables entre elles en début de $I^{\text {er }}$ cycle, mais celles des ray-grass sont supérieures à celles des autres graminées $(\mathrm{P}<0,05)$ à la fin du premier cycle. Enfin, les repousses ont en moyenne une ingestibilité plus élevée que les fourrages du premier cycle de digestibilité comparable, mais les différences ne sont significatives $(P<0,05)$ que pour le brome et le trèfle violet en I97I.

Quantité ingérée lors des repas suivant les distributions de fourrage.

Les quantités de matière sèche ingérées lors des grands repas (qi, moyenne pour les repas du matin et du soir) varient de $\mathbf{r} 6$ à $29 \mathrm{~g} / \mathrm{kg} \mathrm{P}^{0,75}$ pour l'ensemble des graminées et seulement de 27 à $33 \mathrm{~g}$ pour la luzerne et le sainfoin. Ellles sont plus élevées

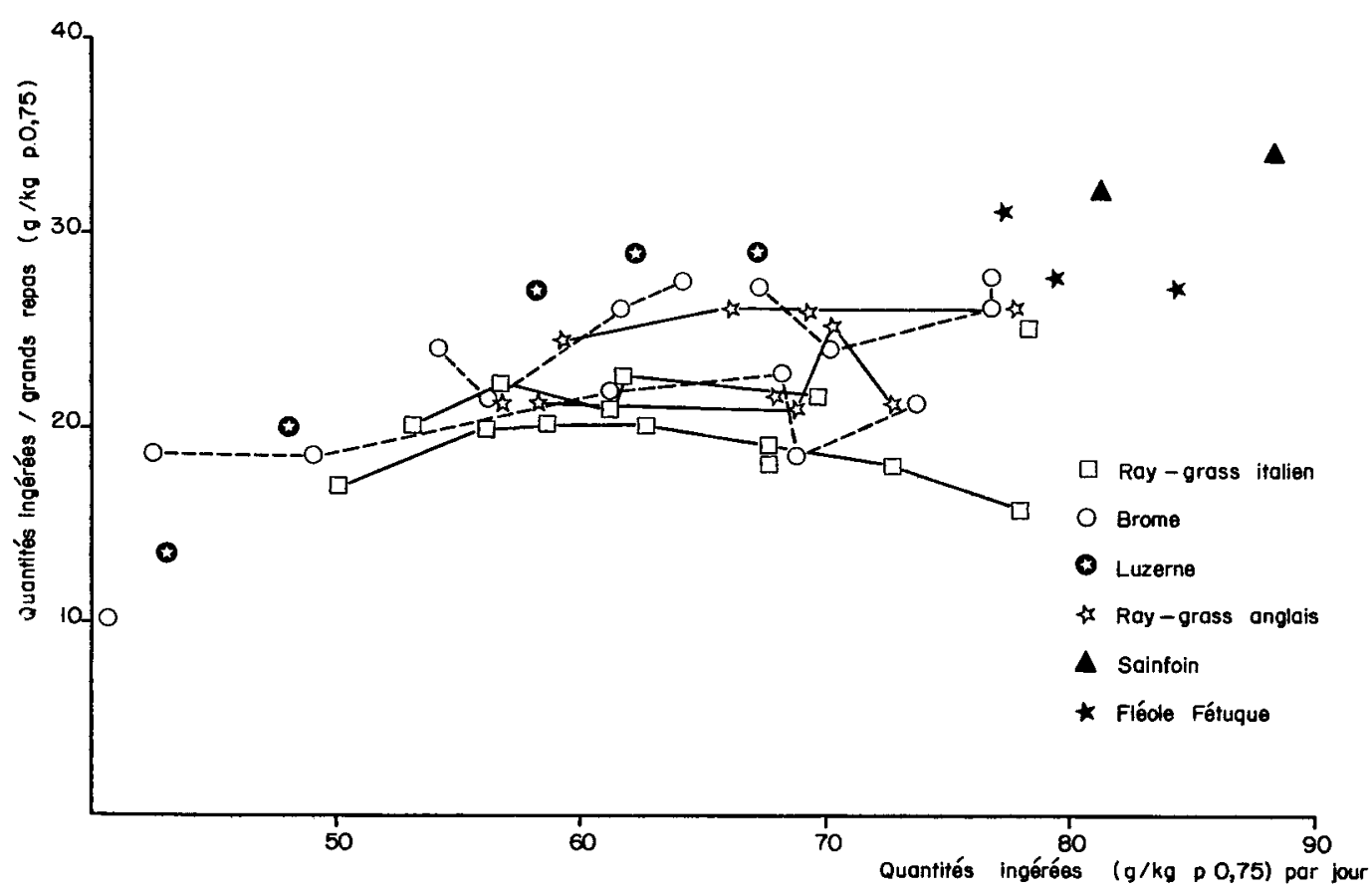

FIG. I. - Relation entre les quantités ingêrées par jour et celles ingérees par grand pepas 
pour les repousses que pour les premiers cycles, quoique les différences ne soient significatives que pour le brome, et sont plus élevées pour la luzerne et le sainfoin que pour les graminées.

Si on rapporte ces quantités à celles ingérées par jour, on note un pourcentage très variable en général compris entre 25 et $45 \mathrm{p}$. Ioo. Ce pourcentage est plus faible pour le ray-grass italien, surtout au premier cycle; pour une espèce donnée il augmente lorsque la plante vieillit.

Pour une plante donnée les quantités ingérées lors des grands repas varient peu en fonction du stade de végétation (fig. 1). İn regroupant tous les échantillons étudiés, nous constatons de même que les variations des quantités ingérées par grands repas ne sont liées ni aux variations des teneurs en matière sèche, en matières azotées totales et en cellulose brute, ni aux variations de digestibilité. Les différences qui existent entre espèces végétales sont, par contre, liées aux quantités ingérées par jour $(Q I)$ :

ou

$$
\begin{aligned}
\mathrm{qi} & =\mathrm{II}, 6+0,165 \mathrm{QI}\left(r=0,443^{* *}\right) \\
\mathrm{QI} & =26,3+\mathrm{I}, 7 \mathrm{I} 6 \mathrm{qi} \quad\left(r=0,662^{* *}\right) .
\end{aligned}
$$

Quant aux quantités de matière fraîche ingérées par grands repas $(85 \mathbf{5}$ à $260 \mathrm{~g} / \mathrm{kg}$ $\mathrm{P}^{0,75}$ ) elles sont maximales lorsque les quantités de matière sèche correspondantes sont maximales et elles diminuent en fin de cycle. Filles dépendent en premier lieu des variations de la teneur en matière sèche du fourrage, surtout pour les graminées, et de l'espèce végétale.

\section{Comportement alimentaire des animaux (tabl. 2)}

\section{Durée journalière d'ingestion.}

Pour l'ensemble des fourrages étudiés, la durée journalière d'ingestion est en moyenne de $3 \mathbf{I} 2$ minutes $( \pm 4 \mathbf{I}$ ). Iille diminue nettement au cours du premier cycle de végétation (de 300-350 à 200-250 minutes) pour le brome et le trèfle violet alors qu'elle évolue assez peu pour les ray-grass et la fétuque (300-350 minutes). Au $2^{\mathbf{e}}$ cycle, il n'y a une diminution importante avec 1'âge que pour le brome et le ray-grass italien, peut-être à cause de la remontée en tiges de ces plantes. Il n'y a pas de différences systématiques entre graminées et légumineuses de même qu'entre cycles de végétation d'une même espèce.

Nombre de repas par jour.

Le nombre de repas par jour est en moyenne de $7, \mathrm{I}( \pm 2,3)$ (variations de 3 à $\mathrm{I}_{5}$ ). Il varie dans le même sens que la digestibilité de la matière organique $\left(r=+0,488^{* *}\right)$ et la teneur en matières azotées totales $\left(r=+0,243^{*}\right)$ et en sens inverse de la teneur en cellulose brute $\left(r=-0,47^{* *}\right)$, cela pour 1'ensemble des échantillons de fourrage sauf la luzerne et le sainfoin. De plus, pour les graminées il augmente lorsque la teneur en matière sèche de la plante diminue $\left(r=-0,640^{* *}\right)$. Le coefficient de corrélation multiple liant les 3 premiers facteurs au nombre de repas n'est cependant que de 0,60 , peut-être en partie à cause de la très grande variabilité du nombre de repas d'un animal à l'autre dans un lot (coefficient de variation de 24 p. IOo).

A même digestibilité le nombre de repas pour la luzerne et le ray-grass d'Italie au premier cycle est supérieur $(\mathrm{P}<0,05)$ à celui des autres plantes (fig. 2), sauf à 


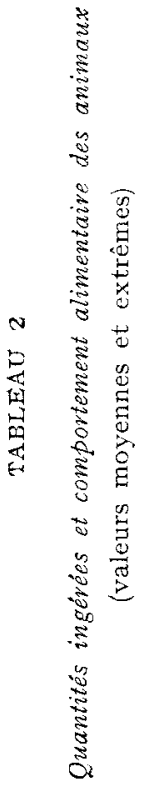

\begin{tabular}{|c|c|c|c|c|c|c|c|c|}
\hline 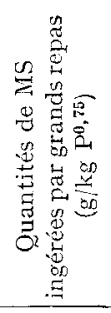 & 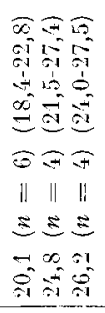 & 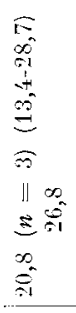 & $\begin{array}{l}= \\
\| \\
\equiv 1 \\
\Xi 1 \\
0 \\
8 \\
8\end{array}$ & 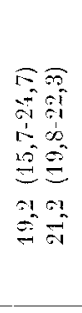 & 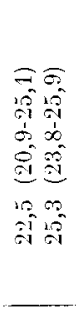 & 11 & 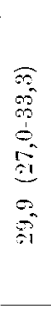 & 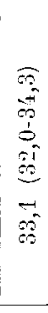 \\
\hline 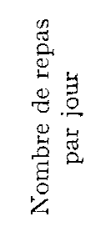 & 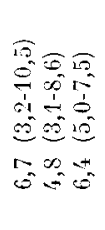 & 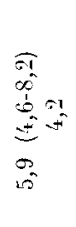 & 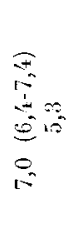 & 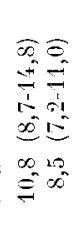 & 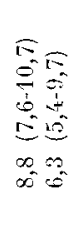 & 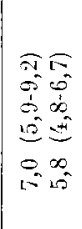 & 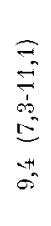 & $\begin{array}{l}0 \\
0 \\
5 \\
0 \\
0 \\
0 \\
2 \\
2\end{array}$ \\
\hline 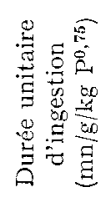 & 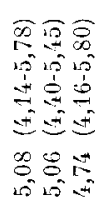 & 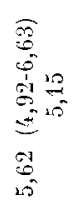 & 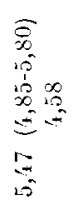 & 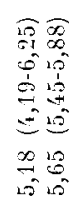 & 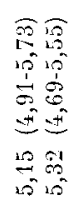 & 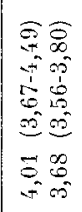 & 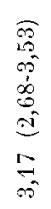 & $\begin{array}{c}\sigma \\
3 \\
1 \\
0 \\
0 \\
0 \\
0 \\
0 \\
0 \\
-1\end{array}$ \\
\hline 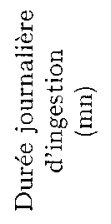 & 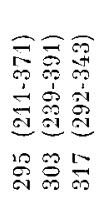 & 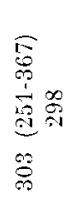 & 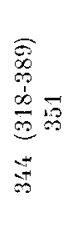 & 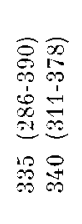 & 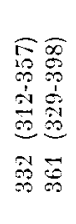 & 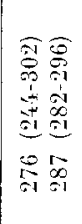 & 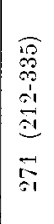 & 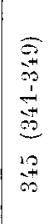 \\
\hline 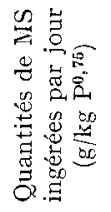 & 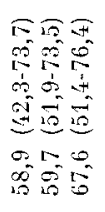 & 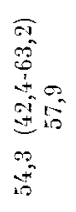 & $\begin{array}{l}0 \\
0 \\
0 \\
0 \\
0 \\
0 \\
0 \\
0 \\
0 \\
8 \\
0 \\
0\end{array}$ & 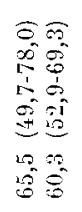 & 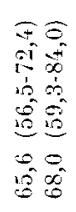 & 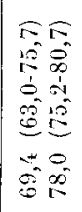 & 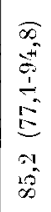 & 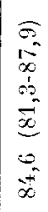 \\
\hline$\approx$ & $\stackrel{\infty}{\rightarrow} \infty$ & $r-$ & $\infty r$ & $\sigma 20$ & e & $\cdots$ & 10 & $\theta$ \\
\hline & 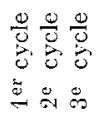 & 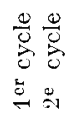 & $\begin{array}{l}0 \\
0 \\
0 \\
0 \\
0 \\
5 \\
5\end{array}$ & 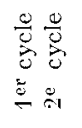 & $\begin{array}{l}\frac{0}{0} \\
0 \\
0 \\
0 \\
5 \\
5\end{array}$ & $\begin{array}{l}0 \\
0 \\
0 \\
0 \\
0 \\
0 \\
\vdots \\
\vdots\end{array}$ & & \\
\hline & 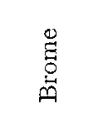 & 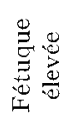 & 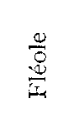 & 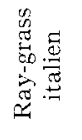 & 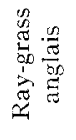 & 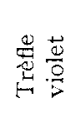 & 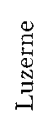 & $\begin{array}{l}\stackrel{\Xi}{0} \\
\stackrel{\Xi}{\Xi} \\
\stackrel{\Xi}{\pi}\end{array}$ \\
\hline
\end{tabular}


ceux du ray-grass anglais en fin de cycle. Il n'y a pas de différence entre $I^{\text {er }}$ et $2^{\circ}$ cycles sauf pour le ray-grass anglais.

Pour les graminées, le nombre de repas augmente lorsque la quantité ingérée par grands repas diminue $\left(r=-0,5 \mathrm{I} 4^{* *} ; n=43\right)$. De plus, le nombre de repas et les quantités ingérées par jour varient dans le même sens pour l'ensemble des fourrages du $I^{\text {er }}$ cycle $(r=0,687)$ et dans une moindre mesure pour les repousses.

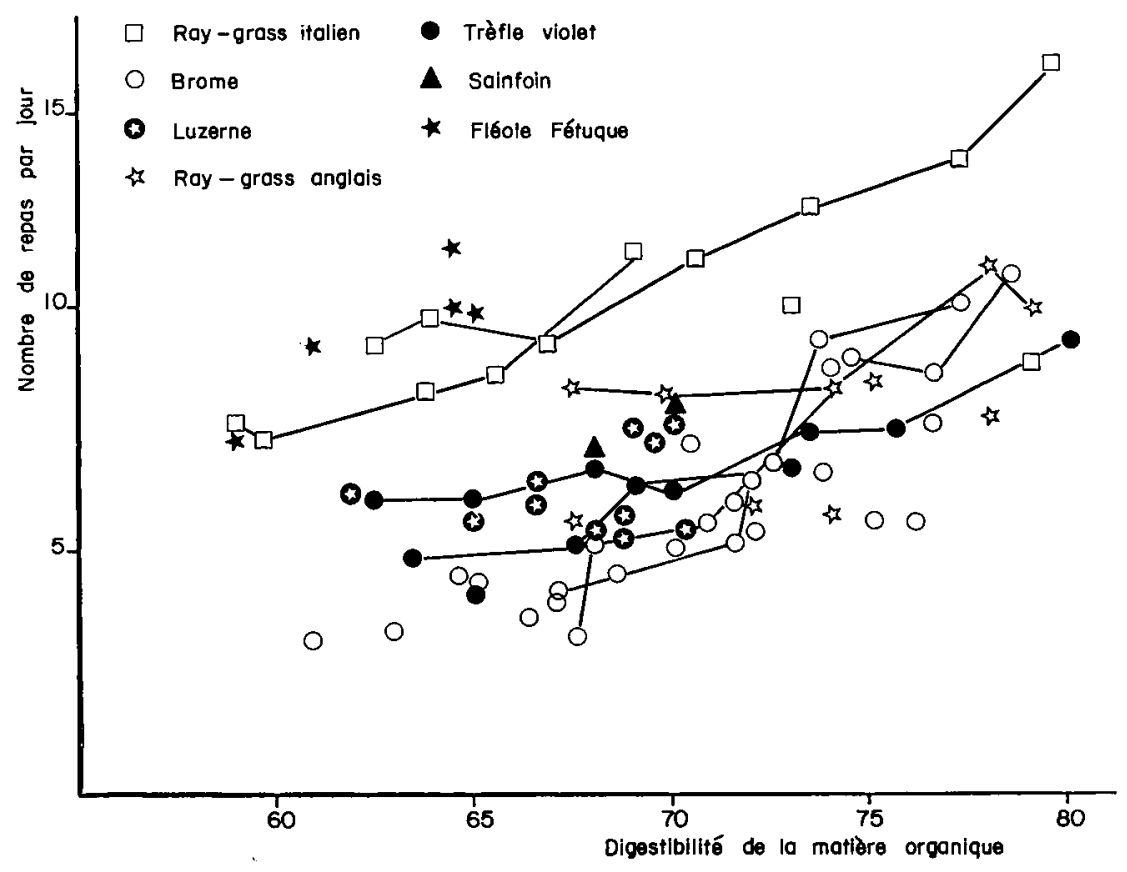

FIG. 2. - Évolution du nombre de repas par jour avec la digestibilité de la matiore organique

Durée unitaire d'ingestion (tabl. 2).

La durée unitaire d'ingestion des fourrages est en moyenne de $5,23 \pm 0,56 \mathrm{mn} / \mathrm{g} / \mathrm{kg}$ $\mathbf{P}^{0,75}$ (4 à 6$)$ pour les graminées et de $3,7 \mathrm{I} \pm 0,44(3$ à 4,5) pour les légumineuses. Eille ne présente pas toujours de variation nette lorsque la plante vieillit. Cependant elle augmente en général durant le premier cycle de végétation. Ainsi, pour le premier cycle de brome étudié en 1972 elle passe de 4,2 à $5,6 \mathrm{mn} / \mathrm{g} / \mathrm{kg} \mathrm{P} \mathrm{P}^{0,75}$, pour le premier cycle de ray-grass italien de 4,6 à 6,0 (les différences début-fin de cycle étant significatives dans ces 2 cas), et pour le ray-grass anglais au ${ }^{\text {er }}$ cycle de 5 à 5,5 (variation non significative). Dans les autres cas on observe peu de variations. L,es durées unitaires d'ingestion ne sont pas significativement différentes d'une graminée à l'autre. En revanche celles des légumineuses sont significativement $(\mathrm{P}<0,05)$ inférieures à celles des graminées et parmi les légumineuses celles de la luzerne sont inférieures à celles du trèfle et du sainfoin.

La durée unitaire d'ingestion au cours des grands repas qui suivent les distributions de fourrage augmente très peu entre le début et la fin du cycle, sauf pour le brome. Elle varie de 4 à $4,5 \mathrm{mn} / \mathrm{g} / \mathrm{kg} \mathrm{P} \mathrm{P}^{0,75}$ pour les graminées et on n'observe pas de différence entre espèce ou cycle de végétation. Avec les légumineuses ces durées uni- 
taires sont de 3,6 pour le sainfoin et 2,5 pour la luzerne. Les différences entre graminées, sainfoin et luzerne sont toutes significatives $(P>0,05)$. Au cours des autres petits repas ultérieurs la durée unitaire d'ingestion est, sauf cas particulier, toujours plus élevée qu'au cours des grands repas.

\section{Comportement merycique des animaux (tabl. 3)}

\section{Durée joumalière de rumination.}

La durée journalière de rumination varie d'environ 400 à 600 minutes par jour et reste comprise entre 500 et 600 minutes pour les plantes les plus âgées. Elle ne présente pas de différences, à même digestibilité, entre espèces végétales ou cycles de végétation.

La durée de rumination est liée à la teneur en cellulose brute de la plante : $\mathrm{R}=0,55^{*}$ pour un ajustement curvilinéaire avec l'ensemble des échantillons étudiés et $\mathrm{R}=0,667^{*}$ pour un même ajustement avec les seuls échantillons du $\mathrm{I}^{\mathrm{er}}$ cycle. Elle est encore plus étroitement liée à la digestibilité de la matière organique $\left(r=-0,769^{* *}\right.$ et $r=-0,736^{* *}$ dans les mêmes conditions).

Il s'agit là de liaisons globales, mais on peut graphiquement mettre en évidence trois populations d'échantillons (fig. 3) :

a) les 5 luzernes,

b) les graminées et les légumineuses autres que la luzerne dont la digestibilité est supérieure à 73 ,

c) les graminées et les légumineuses (sauf luzerne) dont la digestibilité est inférieure à 73 .

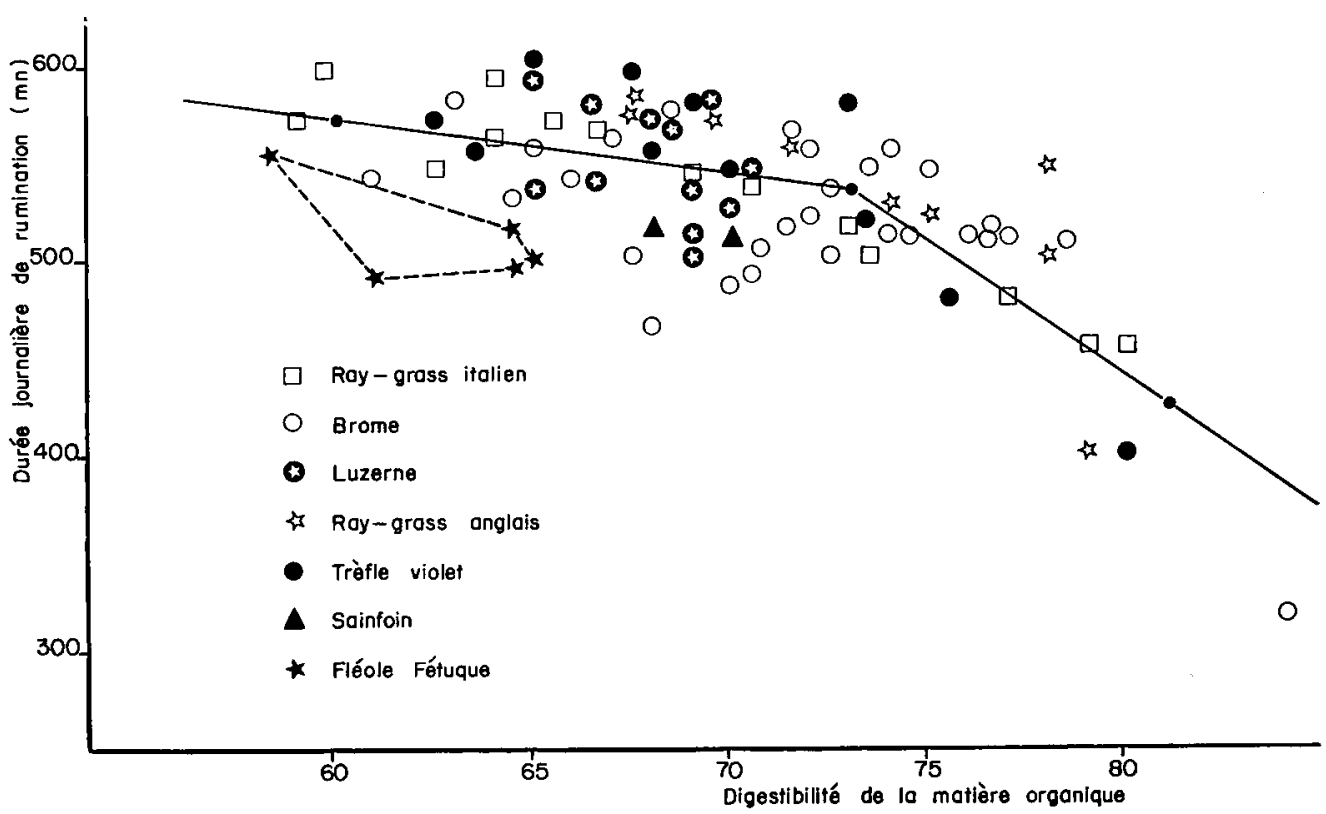

FIG. 3. - Évolution de la durée journalière de rumination avec la digestibilité de la matière organique 


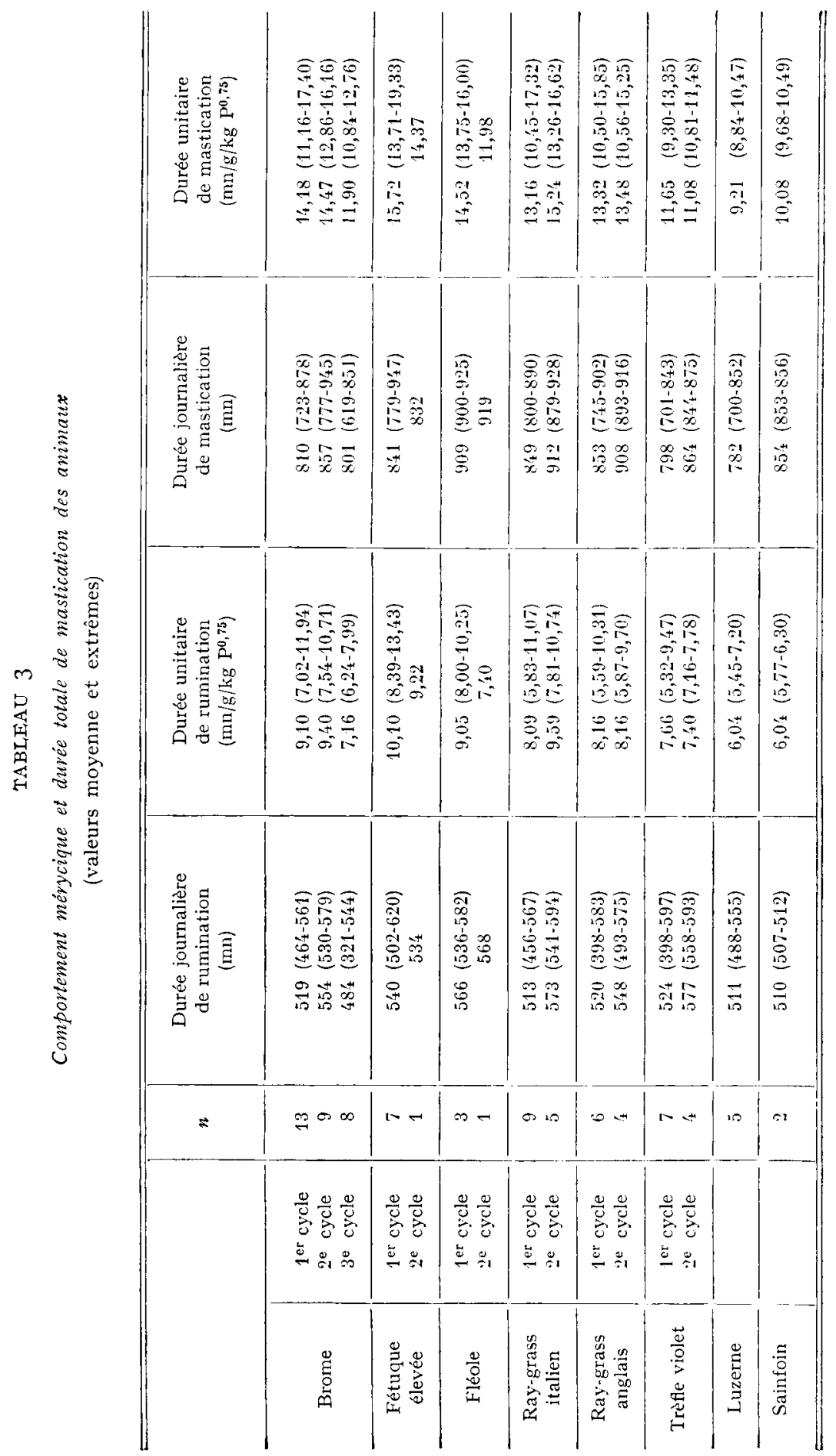


Pour les 5 luzernes la durée de rumination est seulement de 5 Ir minutes pour une digestibilité moyenne de 62,6 , mais cette valeur n'est pas significativement différente de celle des graminées de même digestibilité ( 560 minutes). Pour la population $b$, les variations de la digestibilité de la matière organique expliquent nettement les variations de la durée de rumination; celle-ci diminue de 13,75 minutes par point de digestibilité $\left(r=-0,73^{* *}\right)$. Innfin, pour la population $c$, la durée de rumination varie peu avec la digestibilité de la matière organique, elle ne diminue que de $2,92 \mathrm{mi}$ nutes par point de digestibilité $\left(r=-0,325^{*}\right)$. Pour ces fourrages de digestibilité inférieure à 73 l'écart-type de la moyenne de la durée de rumination d'un fourrage à l'autre est faible (30 minutes) par rapport à l'écart-type pour la durée moyenne de rumination d'un mouton à l'autre ( 52 minutes).

Les liaisons avec la teneur en cellulose brute sont plus faibles que celles trouvées avec la digestibilité de la matière organique : $r=0,372$ pour la population $b$ et $r=0, \mathrm{I} 43$ pour la population $c$.

\section{Durée unitaire de rumination.}

La durée unitaire de rumination est un paramètre très variable (de j à

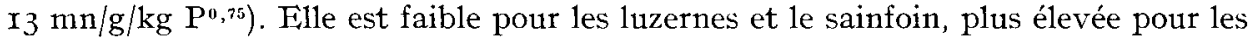
graminées et le trèfle violet. Elle augmente toujours nettement lorsque la plante vieillit. Flle est, pour les graminées et le trèfle, très étroitenlent liée à la teneur en matières azotées totales de l'échantillon $\left(r=-0,723^{* *}\right)$, à sa teneur en cellulose brute $\left(r=+0,772^{* *}\right)$ et à sa digestibilité $\left(r=-0,766^{* *}\right)$.

A même teneur en matières azotées, les durées unitaires de rumination sont comparables pour toutes les espèces végétales. Par contre, à même digestibilité, elles sont nettement inférieures $(\mathrm{P}<0,05)$ pour la luzerne et le sainfoin.

Si on ne considère que les échantillons du premier cycle (graminées et trèfle) on note des liaisons encore plus étroites $\left(r=+0,8 \mathrm{I} 5^{* *}\right.$ avec la teneur en cellulose brute et $r=-0,85^{* *}$ avec la digestibilité de la matière organique). Au second cycle on note également une liaison étroite avec la teneur en matières azotées totales $\left(r=-0,857^{* *}\right)$.

\section{Mastication}

\section{Durée journalière de mastication.}

Le temps journalier passé par les moutons à mastiquer est le paramètre le plus constant du comportement alimentaire : en moyenne 850 minutes $( \pm 59)$ pour les graminées et $8 \mathrm{I} 4$ ( \pm 55 ) pour les légumineuses (différence non significative), la plupart des valeurs étant comprises entre 800 et 900 minutes par jour. L'évolution de cette durée avec l'âge de la plante est variable : elle augmente d'environ roo minutes entre le début et la fin du premier cycle avec les ray-grass, la fétuque et le trèfle car pour ces plantes, l'augmentation de la durée de rumination est importante et la baisse de la durée d'ingestion est faible. Dans le même temps elle diminue avec l'âge du brome au premier cycle car pour cette plante la durée d'ingestion diminue nettement alors que la durée de rumination n'augmente pratiquement pas (durée élevée dès le début du cycle). On n'observe pas d'évolution nette au cours des $2^{\mathrm{e}}$ cycles. La durée de mastication est légèrement plus élevée pour les $2^{\mathrm{e}}$ cycles que pour les premiers par suite principalement d'une augmentation de la durée de rumination, mais ces diffé- 
rences ne sont pas significatives. Pour l'ensemble des fourrages du premier cycle nous avons figuré cette évolution sur la figure 4 .

Notons aussi, et ceci est très important, qu'il n'y a pas de liaison significative entre la durée journalière de mastication et les quantités de matière sèche ingérées.

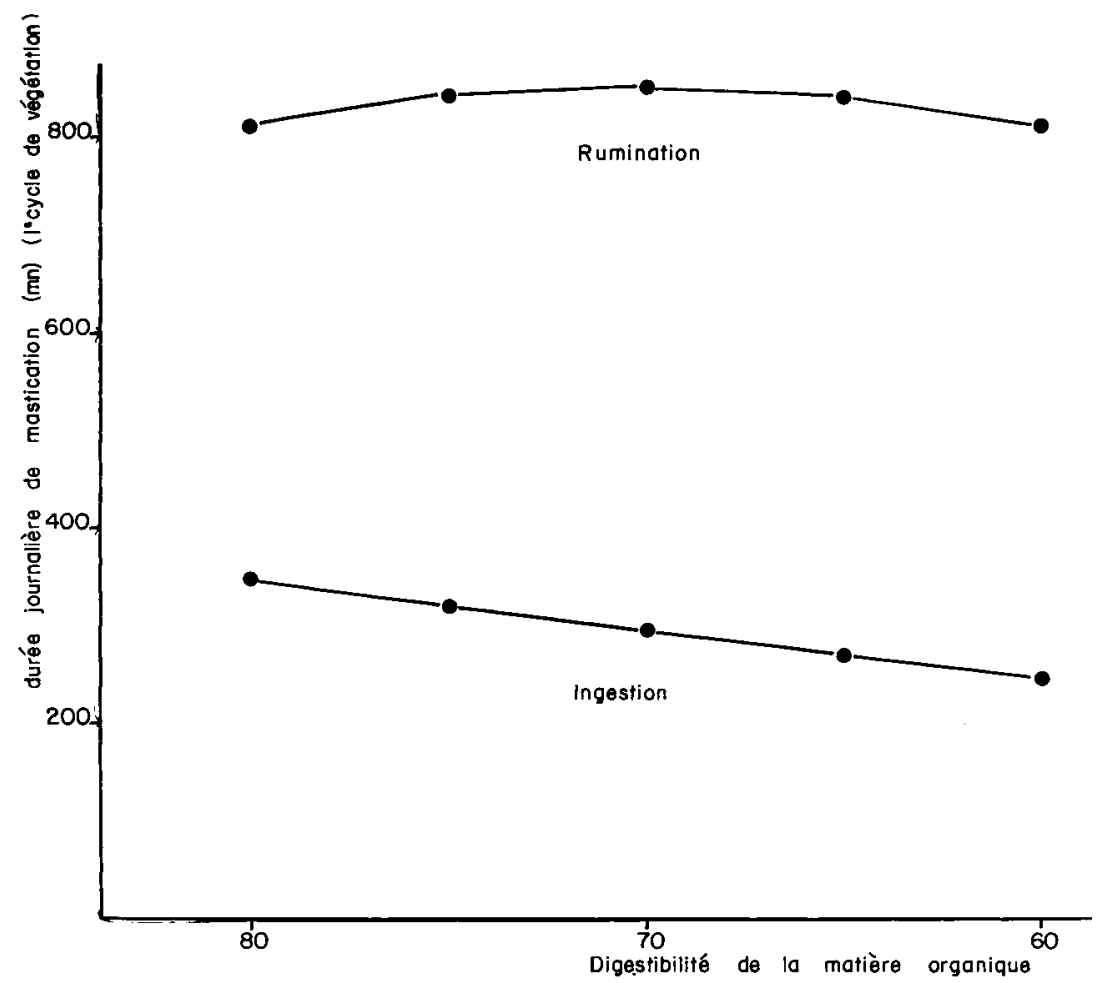

FIr. 4. - Evolution de la durée journaliere de mastication arec la digestibilité de la maticre orgunique

Durée unitaire do mastication.

La durée unitaire de mastication est un paramètre très variable (de 8,8 à $\left.\mathrm{I} 9,3 \mathrm{mn} / \mathrm{g} / \mathrm{kg} \mathbf{P}^{\mathbf{0}, 75}\right)$. Elle est liée étroitement à la teneur en matières azotées de la plante $\left(r=-0,720^{* *}\right)$, à sa teneur en cellulose brute $\left(r=+0,779^{* *}\right)$ et à la digestibilité de sa matière organique $\left(r=-0,687^{* *}\right)$.

A même teneur en matières azotées les durées unitaires de mastication pour la luzerne sont comparables à celles pour les graminées et le trèfle violet.

C'est avec les quantités de matière sèche ingérées par jour QI que les durées unitaires de mastication DUM (fig. 5) sont les plus étroitement liées (DUM $=26,68-2,07 \mathrm{QI} ; r=-0,923^{* *}$ ) pour l'ensemble graminées-trèfle violet, $\left(r=-0,945^{* *}\right.$ au premier cycle et $r=-0,956^{* *}$ au $2^{\mathrm{e}}$ cycle). De ce point de vue, la luzerne est comparable aux autres espèces. 


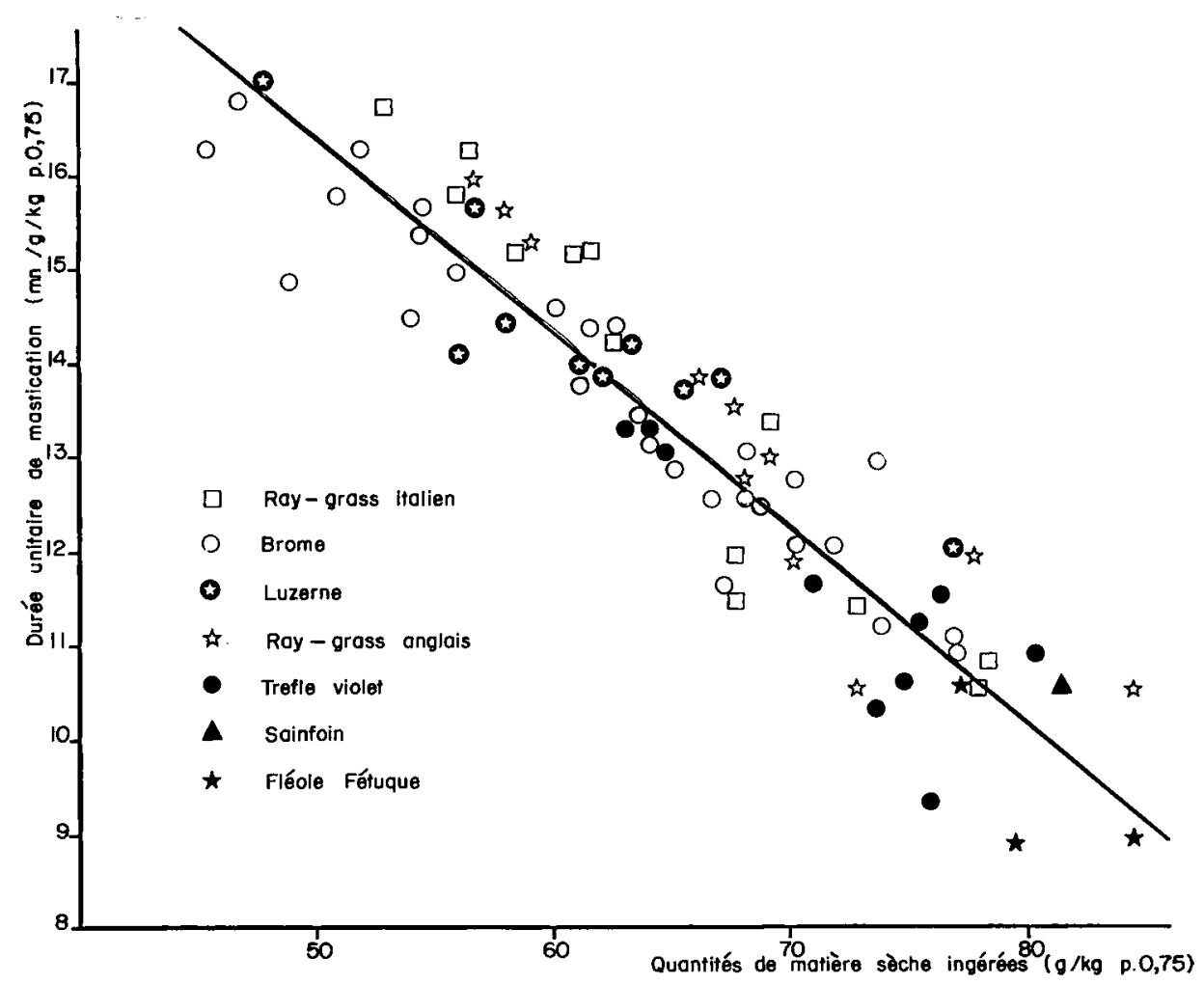

Fig. 5. - Relation entre les quantités journalièrés ingérées et la durée unitairt de mastication

\section{DISCUSSION}

Dans notre première étude sur le comportement alimentaire et mérycique des moutons recevant des fourrages verts (DULPHY et DEMARQUILIY, I974) nous avous principalement discuté le comportement mérycique et le nombre de repas par jour. Les résultats de cette seconde étude confirment ceux de la première, en particulier pour ce qui concerne le nombre de repas par jour. Ils permettent aussi de préciser l'évolution de la durée journalière de rumination. Ils apportent enfin des éléments nouveaux, en particulier pour les quantités ingérées par grands repas et pour expliquer des différences de comportement entre moutons recevant des fourrages d'espèces différentes. L'analyse du comportement alimentaire et mérycique permet donc d'éclairet les mécanismes qui contrôlent la quantité de fourrage ingérée et ses variations au cours du cycle de végétation et avec l'espèce végétale.

La très faible variation des quantités de matière sèche ingérées par grands repas tout au long d'un cycle de végétation d'une même plante est une observation intéressante. Les quantités ingérées par grands repas sont vraisemblablement liées à la quantité de contenu digestif au début du repas (CAMP'ING et al., I96I) à la vitesse de 
passage du contenu du rumen vers le feuillet au cours du repas, à la quantité de contenu digestif à la fin du repas et aussi à la teneur en eau du fourrage ingéré (VÉRIT́́ et Journeit, I970). Pour mieux comprendre le pourquoi du phénomène observé il aurait donc fallu mesurer les contenus de rumen avant et après chacun des grands repas.

Compte tenu de la constance des quantités ingérées par grand repas, les quantités de matière sèche ingérées par jour sont liées principalement au nombre de petits repas effectués par l'animal dans la journée. Comme nous l'avions remarqué précédemment (Dulphy et Demarquir.ty, I974) il diminue avec l'âge de la plante en même temps que la teneur en cellulose brute augmente et que la digestibilité de la plante diminue, c'est-à-dire lorsque la teneur en membranes augmente.

D'une plante à l'autre, à même digestibilité, le nombre total de repas par jour est comparable (environ 9 au début du cycle et 6 à la fin du cycle) sauf pour la luzerne et le ray-grass d'Italie où il est plus élevé. Il est vraisemblable que le nombre de repas est lié à la vitesse de vidange du rumen, donc à la vitesse de digestion du fourrage. Cela peut expliquer le nombre de repas plus élevé avec la luzerne mais non avec le ray-grass d'Italie, du moins quand on le compare avec le trèfle violet. Il est possible que pour le ray-grass d'Italie l'eau contenue dans le fourrage encombre momentanément le rumen et conduit à un nombre élevé de repas. Pour le trèfle, qui est encore plus riche en eau que le ray-grass d'Italie, il est possible que l'importance des quantités ingérées par grands repas limite le nombre de petits repas. Ces deux hypothèses nous sont suggérées par les relations négatives qui existent entre nombre de repas par jour d'une part et la teneur en matière sèche de la plante et les quantités ingérées par grands repas d'autre part.

L'augmentation de la durée journalière de rumination au cours du vieillissement des plantes est nette. Comme l'indiquent les liaisons trouvées avec la digestibilité et la teneur en cellulose brute, cette augmentation est liée surtout à la teneur en membranes. La durée de rumination augmente d'abord rapidement lorsque la digestibilité de la plante diminue, puis lentement sans dépasser 600 minutes par jour. La rumination étant nécessaire à la réduction du fourrage en particules suffisamment fines pour quitter le rumen, il semble donc que la durée de rumination puisse devenir un facteur limitant de la quantité ingérée. La durée maximale de rumination qui est située vers 550-600 minutes par jour pourrait alors être une caractéristique de l'animal car elle est observée pour des échantillons très différents de fourrage.

Nous n'avons jamais observé des durées de rumination égales à 650-700 minutes comme l'a observé GORDON (I965) en augmentant progressivement les quantités de foin offertes aux animaux. Il est vraisemblable que ces durées élevées étaient liées au fait que les moutons avaient été très fortement rationnés durant le début de 1'expérience. $\mathrm{L}_{4}$ a limite de 550-600 minutes que nous observons avec des fourrages verts correspond d'ailleurs à celle que trouve XANDE (communication personnelle), $540 \mathrm{mn}$ avec des rations à base de pailles seules ou complémentées ayant une digestibilité comprise entre 40 et 60 .

De même la durée journalière de mastication ne dépasse guère goo mn par jour et les liaisons très étroites qui existent entre les quantités ingérées et la durée unitaire de mastication correspondent à celles qu'ont trouvé SkourI (I969) et BAL_H (I97I) avec des vaches laitières. Leur existence semblerait montrer que le premier facteur qui explique les variations de quantités ingérées est le temps nécessaire pour masti- 
quer une quantité unitaire de fotrrage, ce temps étant vraisemblablement 1ui-même lié à la teneur en membranes et à la vitesse de digestion de la plante dans le rumen.

In fin de compte, lorsqu'une plante vieillit, on observe chez le mouton qui l'ingère une diminution du nombre de repas, une augmentation de la durée de rumination jusqu'à un plafond et un accroissement très net de la durée unitaire de mastication. Ces phénomènes sont liés à la digestibilité de la plante et à ses teneurs en matières azotées et en cellulose. En fait, il est vraisemblable que le principal facteur soit la variation de la teneur en membranes des plantes, leur teneur en cellulose, leur digestibilité et leur vitesse de digestion n'étant que des conséquences de cette variation. Ensuite, le fait que la durée journalière de mastication varie peu doit alors plus ou moins fixer la quantité ingérée par jour, du moins tant que la régulation de cette quantité ingérée est de type "physique".

Les différences de comportement qui existent entre les espèces végétales doivent donc provenir en premier lieu des différences de teneur en membranes responsables de la digestibilité et de la vitesse de digestion variables de ces plantes. İlles proviennent également de leurs différences de teneur en matières azotées et en eau. Ces trois facteurs induisant des différences au niveau du comportement des animaux entraînent en fin de compte des différences de quantités ingérées.

Resu pour publication en avril 1976.

\section{SUMMARY}

\section{FFFECT OF PLANT SPECIES AND GROWTH STAGE ON FEFDING BEHAVIOUR AND RUMINATION IN SHEEP RECEIVING CHOPPED GREEN FORAGES}

I. The observations made by Dulphy and Demarguiley (I974) have been completed in the present study by investigating the influence of plant species and growth stage on feeding behaviour and rumination in sheep stall-fed with chopped green forages. This study was made with 84 samples of green forages (table 1 ) among which 62 belonged to 4 plant species systematically examined during their different growth cycles.

2. The percentage of dry matter ingested by the animals at each of the 2 meals following the administration of the forage was much variable as compared to the daily dry matter intake ( 25 to $45 \mathrm{p}$. Ioo, table 2 ), but in absolute value, the amounts ingested during these large meals varied little according to the growth stage of the forage (fig. I), i.e. according to its digestibility, voluntary intake and chemical composition. The amounts eaten were much higher for legumes than for grasses.

3. In the sheep, the daily time spent eating generally decreased with the stage of maturity of the plant; the number of meals per day also decreased (fig. 2), whereas the unitary eating time remained constant or increased.

4. The daily time spent ruminating rapidly increased when the digestibility of the plant decreased from 80 to about $73 \mathrm{p}$. Ioo (fig. 3). When the digestibility was below $73 \mathrm{p}$. Ioo, the time spent ruminating increased much slowlier and seemed not to excecd 600 minutes per day. Simultancously, the unitary ruminating time definitely increased when the digestibility of the plant decreased.

5. The daily time spent chewing varied little with the stage of maturity of the plant (fig. 4). On the other hand, the unitary chewing time (UCT) rapidly increased when the quantities ingested (QI) per day decreased (fig. 5) :

$$
\text { (UCT : } 26.68-2.07 Q 1 ; r=-0.923^{* *} \text { ) }
$$

6. The differences observed between plant species were sometimes large, but this could probably be explained by differences between their digestibility as well as dry matter, crude protein and crude fibre contents. 


\section{RÉFÉRENCES BIBLIOGRAPHIQUES}

Balcr C. C., r97r. Proposal to use time spent chewing as an index of the extent to which diets for ruminants possess the physical property of fibrousness characteristic of routhages. $\quad$ Br. J. Nutr., 26, 383 .

Campling R. C., Freel M., Balcil C. C., I06I. lactors affecting the voluntary intake of food by cows (no 2). Br. J. Nutr., 15, 531-540.

DULphy J.-P., I97I. Influence du poids vif et du niveau d'ingestion sur le comportement alimentaire et mérycique du mouton. Ann. Zootech., 20, 477-486.

Ducphy J.-I'., Demaroully $\quad$ C., I973. Influence de la machine de récolte et de la finesse de hachage sur la valeur alimentaire des ensilages. Ann. Zootcch., 22, I99-2I7.

Dulphy J.-P., Dimarquilly C., r974. Fitucle du comportement alimentaire et inérycique de moutons recevant des fourrages verts hachés. Ann. Zootech., 23, I93-212.

Gordon J.G., I965. The relationship between rumination and the amount of roughages eaten by sheep. J. Agric. Sci., 64, I5 $\mathrm{I}^{-1} 55$.

Ruckenusch Y., 1963. Recherches sur la régulation contrale du comportenont alimentaire chez les runinants, Thèse Doct. Sci. Université de Lyon.

Skouri M., I967. Valeur nutritive de la ration et comportement alimentaire dut ruminant. Ann. Inst. Nat. Rech. Agr., Tunisie, 4o, fasc. 6, 152 p.

Verité R., Journet M., I97o. Influence de la teneur en eau et de la déshydratation de l'herbe sur sa valeur alimentaire pour les vaches laitières. Ann. Zootech., 19, 255-268.

Xnnde A., rg76. Valeur alimentaire des pailles de céréale (en préparation). 